\title{
Physical Function and Clinical Outcomes in Hemodialysis Patients: The China Dialysis Outcomes and Practice Pattern Study
}

Qingyu Niu

Peking University People's Hospital

\section{Xinju Zhao}

Peking University People's Hospital

\section{Liangying Gan}

Peking University People's Hospital

\section{Xinling Liang}

Guangdong Provincial People's Hospital

\section{Zhaohui Ni}

Shanghai Jiao Tong University School of Medicine Affiliated Renji Hospital

\section{Xiaonong Chen}

Shanghai Jiao Tong University Medical School Affiliated Ruijin Hospital

\section{Yuqing Chen}

Peking University First Hospital

\section{Fan Fan Hou}

Southern Medical University Nanfang Hospital

Li Zuo ( $\square$ zuoli@bjmu.edu.com )

Peking University People's Hospital https://orcid.org/0000-0002-7340-5995

\section{Research}

Keywords: Physical function, mortality, hospitalization, hemodialysis, DOPPS

Posted Date: May 29th, 2020

DOl: https://doi.org/10.21203/rs.3.rs-31249/v1

License: (c) (i) This work is licensed under a Creative Commons Attribution 4.0 International License. Read Full License 


\section{Abstract}

Background: Hemodialysis (HD) patients usually have impaired physical function compared with general population. Self-reported physical function is a simple method to implement in daily dialysis care. This study aimed to examine the association of self-reported physical function with clinical outcomes of HD patients.

Methods: Dialysis Outcomes and Practice Patterns Study (DOPPS) is a prospective cohort study. Data on 1427 HD patients in China DOPPS 5 were analyzed. Self-reported physical function was characterized by 2 items of 'moderate activities limited level' and 'climbing stairs limited level'. Demographic data, comorbidities, hospitalization and death records were collected from patients' records. Associations between physical function and outcomes were analyzed using COX regression models. The generalized linear mixed models were used to examine correlates of physical function limited level.

Results: Compared to 'limited a lot' in moderate activities, 'limited a little' and 'not limited at all' groups were associated with lower all-cause mortality after adjusted for covariates (HR: 0.652, 95\% Cl: 0.4350.977 and HR: $0.472,95 \% \mathrm{Cl}: 0.241-0.927$, respectively). And not limited in moderate activities was associated with lower risk of hospitalization compared with 'limited a lot' group after adjusted for covariates (HR: $0.747,95 \% \mathrm{Cl}$ : 0.570-0.978). Meanwhile, compared to 'limited a lot' in climbing stairs, 'limited a little' and 'not limited at all' groups were associated with lower all-cause mortality (HR: 0.574, 95\% Cl: $0.380-0.865$ and HR: $0.472,95 \%$ Cl: $0.293-0.762$, respectively) but not hospitalization after fully adjusted. Factors including old age, female gender, longer dialysis vintage, catheter use, low serum albumin and combining with diabetes, coronary artery disease and cerebrovascular disease were related to higher limited levels of physical function $(P<0.05)$.

Conclusion: Higher limited levels in self-reported physical function were associated with higher risk of allcause mortality and hospitalization in HD patients.

\section{Introduction}

Physical function is a widespread measurement tool that most likely represents a composite result of many different physiology and psychosocial factors. Compare with the general population, a reduction of physical function could be observed at patients with early stage of chronic kidney disease (CKD) $[1,2]$, and this condition gets worse and more pronounced in hemodialysis (HD) patients $[3,4]$. Numerous CKD patients suffer from fatigability, muscle wasting, muscle weakness and sarcopenia[5]. Evidence from studies of the general population and CKD patients demonstrated that impaired physical function was strongly associated with increased all-cause mortality and other outcomes[6-9]. The clinical practice guidelines for cardiovascular disease in dialysis patients published by Kidney Disease Outcomes Quality Initiative (K/DOQI) suggested that nephrology and dialysis staff should measure patients' physical function and encourage them to increase physical activity[10]. However, staff in many nephrology departments and dialysis centers didn't routinely assessed patients' physical function, explained the importance of exercise, nor provided measures for preventing the deterioration of physical function [11]. A 
2003 survey indicated that only $38 \%$ of their nephrologists reported frequently assessing patient's physical activity condition and counseling sedentary patients to increase activity[12].

There are a number of methods available to assess physical activity levels, such as gait speed, intermittent shuttle walk test, the 6-minute walk test, peak oxygen uptake and questionnaires[13-15]. Although many tests have been validated in the general population, some of them weren't suitable for dialysis patients. There are many issues that need to be considered in the assessment of the physical function of dialysis patients. For example, dialysis patients are more likely to have sarcopenia then others and they usually have weakness and fluid overload problems[16, 17]. Other considerations include time available for testing since they spend a lot of time lying down or sitting for dialysis treatment every week, suitable location (dialysis center versus laboratory) and staff expertise[11]. These factors may limit the reliability, reproducibility, and prognostic utility of various assessment methods of physical function.

However, self-reported measures, such as Short-Form 12 (SF-12)[18, 19], may offer a more operative, convenient and cost-effective way to assess dialysis patients' physical function. SF-12 is a self-reported questionnaire, which is frequently used to assess health-related quality of life (HRQOL)[20]. SF-12 is shorter than Short-Form 36. Physical component summary (PCS) score and mental component summary (MCS) score can be calculated by using SF-12. In our previous study, we found that lower PCS score was associated with higher all-cause mortality (unpublished data). In SF-12, there are 2 items that represent physical function, namely moderated activities limited level and climbing stairs limited level. They are important basic movements that can be easily assessed as a part of physical function evaluation.

Therefore, we hypothesized that higher limited levels of self-reported physical function (moderated activities and climbing stairs limited level) were associated with a higher risk of mortality and hospitalization among HD patients. We conducted a large prospective cohort study using data from China Dialysis Outcomes and Practices Pattern Study (DOPPS) to investigate whether these 2 simple items of self-reported physical function were associated with mortality and first hospitalization.

\section{Methods}

\section{Study design and subjects}

The DOPPS is an international prospective cohort study of in-center adult HD patients including more than 20 countries in the world, which described in previous published papers[21,22]. The China DOPPS was carries out in the 3 largest cities from the metropolitan areas in China (Beijing, Shanghai and Guangzhou). We randomly selected 15 dialysis facilities in each city and included an average of 30 patients at each facility. Finally, there were 1427 patients participated in China DOPPS5.

Of the 1427 patients, 134 patients were excluded from the present analysis as they didn't answer the questions of self-reported physical function in the patient questionnaire survey. Baseline demographic and clinical data were collected at the start of participation in DOPPS5. 


\section{2 items to assess physical function}

In China DOPPS5, there were 2 items about physical function as a part of SF-12v2 in patient questionnaire survey. The 2 items were 'does your health now limit you in moderate activities, such as moving a table, pushing a vacuum cleaner, bowling, or playing golf? If so, how much?' and 'does your health now limit you in climbing several flights of stairs? If so, how much?'. Both the 2 items had 3 responses options: 1, 'Yes, limited a lot'; 2, 'Yes, limited a little'; 3, 'No, not limited at all'. Patients completed the questionnaire soon after entered study.

\section{Outcomes}

The primary end-point event was all-cause mortality. The secondary end-point event was the first hospitalization with any causes during the follow-up period. Hospitalization event was defined as an inpatient hospitalization with an overnight stay, which was registered in the DOPPS survey. Observation or outpatient records were not included in this analysis.

\section{Statistics Analysis}

Continuous variables were represented as Mean \pm SD or median $\left(25^{\text {th }}, 75^{\text {th }}\right)$ according to the results of normality test. Categorical variables were expressed as percentage. We stratified data according to moderate activity limited level and climbing stairs limited level, respectively. Differences in mean or median among groups were evaluated by using analysis of variance or non-parametric test. And categorical data were compared by using chi-square test.

Based on clinical experience and suggestions from previous published articles, we adjusted for many covariates, which were possibly associated with patient's physical function or outcomes. Covariates in this study included patient's demographic data (age, sex, dialysis vintage, body mass index (BMI) and whether urine output $>200 \mathrm{ml} /$ day), clinical characteristics (serum hemoglobin $(\mathrm{Hgb}$ ) and serum albumin (Alb)), dialysis prescriptions (single-pooled Kt/V, dialysis frequency (whether less than 3 times per week), vascular access type) and comorbidities (diabetes, coronary artery disease, congestive heart failure, other cardiovascular disease, cerebrovascular disease, hepatitis B and C, cancer (non-skin), peripheral vascular disease, lung disease and hypertension).

Survival curves were produced by using the Kaplan-Meier method and estimated by using log-rank test. Association between moderate activity limited level, climbing stairs limited level and all-cause mortality were analyzed using COX regression models. All COX models accounted for facility clustering effects by using the robust sandwich covariance estimate. Survival time for COX models of all-cause mortality was the time from study entry to the end of study or to death. And failure time for COX models of hospitalization was the time from study entry to the end of study or to the first hospitalization. We made 1 unadjusted model and 3 adjusted models for each endpoint. The adjusted covariates included in model 
1: age, gender, vintage; model 2: model 1 variables plus BMI, Hgb, Alb, spKt/V, urine output, vascular access type; model 3: model 2 variables plus comorbidities (diabetes, coronary artery diseases, congestive heart failure, other cardiovascular diseases, cerebrovascular disease, hypertension, peripheral vascular disease, hepatitis B and C, lung disease, cancer).

We also used the generalized linear mixed models (the GLIMMIX procedure) to analyze the associations between the 2 items of physical function and patients' baseline characteristics, as moderate activities limited level and climb stairs limited level were ordinal categorical variables. In these models, we used robust variance estimation to account for facility clustering effects. Adjusted odds ratio (OR) and $95 \%$ conference interval $(\mathrm{Cl})$ were calculated for each variable.

We performed MI procedure to impute missing data, and continuous and categorical variables were imputed by fully conditional specification regression and logistic regression, respectively. After 20 steps of imputation, 20 data sets were combined for the final analysis of Cox regression model and generalized linear mixed model. Percentages of missing for most variables were $<10 \%$, except for single-pooled Kt/V $(36.2 \%)$. $P$ value $<0.05$ was seen as statistically significant. All statistical analyses were performed with SAS, version 9.4 (SAS institute, Cary, NC; USA).

\section{Results}

\section{Demographic data and clinical characteristics}

There were 1233 patients had baseline self-reported moderated activities limited levels. 466 patients (37.8\%) responded that their moderate activities limited levels were 'Yes, limited a lot', 497 (40.3\%) were 'Yes, limited a little' and 270 (21.9\%) were 'No, not limited at all'. Patients with lower self-reported moderate activities level tended to be older and women. They also had a higher possibility of longer dialysis vintage, higher proportion of urine output $<200 \mathrm{ml} /$ day and catheter use, lower serum Alb and $\mathrm{Hgb}$; and with more comorbidities, such as diabetes, coronary artery diseases, congestive heart failure, other cardiovascular diseases, cerebrovascular disease, peripheral vascular disease and lung disease (Table 1). 
Table 1

Baseline characteristics of HD patients according to the moderate activities limited level

\begin{tabular}{|c|c|c|c|c|}
\hline \multirow[t]{2}{*}{ Variables } & \multicolumn{3}{|c|}{ Moderate activities limited level } & \multirow[t]{2}{*}{$P$ value } \\
\hline & $\begin{array}{l}\text { Yes, limited a lot } \\
(n=466)\end{array}$ & $\begin{array}{l}\text { Yes, limited a little } \\
(n=497)\end{array}$ & $\begin{array}{l}\text { No, not limited at all } \\
(n=270)\end{array}$ & \\
\hline \multicolumn{5}{|l|}{ Demographics } \\
\hline Age (years) & $67(55,77)$ & $58(48,67)$ & $53(43,62)$ & $<0.0001^{*}$ \\
\hline Males (\%) & 45.2 & 57.7 & 66.3 & $<0.0001^{*}$ \\
\hline Vintage (years) & $3.1(0.9,6.4)$ & $2.3(0.8,5.0)$ & $2.7(1.0,5.4)$ & $0.0095^{\star}$ \\
\hline BMI & $21.6(19.1,24.1)$ & $21.4(19.4,23.8)$ & $21.5(19.4,23.6)$ & 0.9798 \\
\hline $\begin{array}{l}\text { Urine output } \\
>200 \mathrm{ml} / \text { day }(\%)\end{array}$ & 26.6 & 34.4 & 37.6 & $0.0037 *$ \\
\hline \multirow[t]{2}{*}{$\begin{array}{l}\text { Primary kidney } \\
\text { diseases (\%) }\end{array}$} & & & & - \\
\hline & 33.9 & 43.4 & 57.4 & \\
\hline Glomerulonephritis & 33.9 & 22.4 & 13.7 & \\
\hline nephropathy & 18.7 & 16.5 & 12.5 & \\
\hline $\begin{array}{l}\text { Hypertensive } \\
\text { nephropathy }\end{array}$ & 13.5 & 17.7 & 16.5 & \\
\hline others & & & & \\
\hline
\end{tabular}

\section{Laboratory tests}

\begin{tabular}{lllll}
$\mathrm{Hgb}(\mathrm{g} / \mathrm{dl})$ & $10.4(8.9,11.6)$ & $10.5(9.3,11.7)$ & $10.9(9.6,11.7)$ & $0.0153^{*}$ \\
\hline Alb $(\mathrm{g} / \mathrm{dl})$ & $3.8(3.5,4.1)$ & $4.0(3.8,4.2)$ & $4.1(3.9,4.3)$ & $<0.0001^{*}$ \\
$\begin{array}{l}\text { White blood cells } \\
\left(10^{\wedge} 9 / \mathrm{L}\right)\end{array}$ & $5.9(4.9,7.2)$ & $6.0(5.0,7.2)$ & $6.0(4.7,7.2)$ & 0.8801
\end{tabular}

\section{Dialysis prescription}

\begin{tabular}{lllll|}
\hline spKt/V & $1.3(1.2,1.5)$ & $1.4(1.2,1.5)$ & $1.3(1.1,1.5)$ & 0.2035 \\
$\begin{array}{l}\text { Dialysis <3 times } \\
\text { /week (\%) }\end{array}$ & 19.8 & 21.2 & 25.2 & 0.2218 \\
\hline Fistula use (\%) & 78.2 & 90.1 & 94.3 & $<0.0001^{*}$ \\
\hline $\begin{array}{l}\text { Comorbidities (\%) } \\
\text { Diabetes }\end{array}$ & 36.2 & 25.2 & & $<0.0001^{*}$ \\
$\begin{array}{l}\text { Coronary artery } \\
\text { disease }\end{array}$ & 37.5 & 22.4 & 15.8 & $<0.0001^{*}$ \\
\hline
\end{tabular}




\begin{tabular}{|lcccc|}
$\begin{array}{l}\text { Congestive heart } \\
\text { failure }\end{array}$ & 31.5 & 19.4 & 14.5 & $<0.0001^{*}$ \\
\hline $\begin{array}{l}\text { Other cardiovascular } \\
\text { disease }\end{array}$ & 27.5 & 17.9 & 12.3 & $<0.0001^{*}$ \\
$\begin{array}{l}\text { Cerebrovascular } \\
\text { disease }\end{array}$ & 22.6 & 11.7 & 4.5 & $<0.0001^{*}$ \\
\hline $\begin{array}{l}\text { Hypertension } \\
\begin{array}{l}\text { Peripheral vascular } \\
\text { disease }\end{array}\end{array}$ & 84.4 & 88.4 & 85.8 & 0.1961 \\
\hline Hepatitis & 14.0 & 5.9 & 3.7 & $<0.0001^{*}$ \\
\hline Lung disease & 8.0 & 14.1 & 13.0 & 0.6871 \\
\hline Cancer (non-skin) & 4.8 & 3.6 & 1.9 & $0.0003^{*}$ \\
\hline
\end{tabular}

Note: BMI, body mass index; Hgb, hemoglobin; Alb, albumin; spKt/V, single-pooled Kt/V.

Meanwhile, data on self-reported climbing stairs limited level were available for 1254 patients. 339 patients $(27.0 \%)$ responded that their climbing stairs limited levels were 'Yes, limited a lot', 471 (37.6\%) were 'Yes, limited a little' and 440 (35.1\%) were 'No, not limited at all'. Similar to the results of moderate activities limited level, patients reported lower ability of climbing stairs tended to be older and women. They also had a higher possibility of higher proportion of urine output $<200 \mathrm{ml} /$ day and catheter use, lower serum Alb and Hgb; and with more comorbidities, such as diabetes, coronary artery diseases, congestive heart failure, other cardiovascular diseases, cerebrovascular disease, peripheral vascular disease and lung disease (Supplementary table 1).

\section{Associations between moderated activities limited level and outcomes}

Among 1233 patients who reported moderated activities limited levels, 166 (1.3\%) died and 552 (44.8\%) had hospitalization records during the follow-up period. According to the results of Kaplan-Meier analysis, patients with higher limited levels of moderate activities had significant higher risk of all-cause mortality and hospitalization (log-rank test, $\mathrm{P}<0.0001$, Fig. $1 \mathrm{~A}$ and B). In fully adjusted COX model, 'No, not limited at all' and 'Yes, limited a little' in moderate activities were associated with decreased risk of all-cause mortality compared with 'Yes, limited a lot' group (HR: 0.472, 95\% Cl:0.241-0.927 and HR: $0.652,95 \% \mathrm{Cl}$ : 0.435-0.977, respectively) (Table 2, Fig. 2A). After adjusting for all the covariates, 'no limited' in moderate activities was negatively associated with hospitalization (HR: $0.747,95 \% \mathrm{Cl}: 0.570-0.978$ ) compared with 'limited a lot' group, but 'a little limited' group wasn't shown a significantly negative relationship with hospitalization (HR: 0.954, 95\% Cl: 0. 753-1.207) (Table 3, Fig. 2B). 
Table 2

Associations between moderate activities limited level and all-cause mortality in different COX regression models.

\begin{tabular}{|lllll}
\hline & $\begin{array}{l}\text { Unadjusted } \\
\text { model } \\
\text { HR }(95 \% \mathrm{Cl})\end{array}$ & $\begin{array}{l}\text { Adjusted model 1 } \\
\mathrm{HR}(95 \% \mathrm{Cl})\end{array}$ & $\begin{array}{l}\text { Adjusted model 2 } \\
\mathrm{HR}(95 \% \mathrm{Cl})\end{array}$ & $\begin{array}{l}\text { Adjusted model 3 } \\
\text { HR }(95 \% \mathrm{Cl})\end{array}$ \\
$\begin{array}{l}\text { Moderate activities limited } \\
\text { level }\end{array}$ & & & \\
$\begin{array}{l}\text { Yes, limited a } \\
\text { lot }\end{array}$ & Ref. & Ref. & Ref. & Ref. \\
\hline $\begin{array}{l}\text { Yes, limited a } \\
\text { little }\end{array}$ & $\begin{array}{l}0.408(0.300- \\
0.555)\end{array}$ & $0.549(0.382-0.780)$ & $0.615(0.414-0.916)$ & $0.652(0.435-0.977)$ \\
\hline $\begin{array}{l}\text { No, not } \\
\text { limited at all }\end{array}$ & $\begin{array}{l}0.229(0.119- \\
0.439)\end{array}$ & $0.355(0.183-0.687)$ & $0.414(0.212-0.810)$ & $0.472(0.241-0.927)$ \\
\hline
\end{tabular}

\section{Climbing stairs limited level}

\begin{tabular}{lllll}
$\begin{array}{l}\text { Yes, limited a } \\
\text { lot }\end{array}$ & Ref. & Ref. & Ref. & Ref. \\
$\begin{array}{lllll}\text { Yes, limited a } \\
\text { little }\end{array}$ & $\begin{array}{l}0.380(0.265- \\
0.545)\end{array}$ & $0.480(0.329-0.701)$ & $0.530(0.356-0.787)$ & $0.574(0.380-0.865)$ \\
\hline $\begin{array}{l}\text { No, not } \\
\text { limited at all }\end{array}$ & $\begin{array}{l}0.229(0.150- \\
0.348)\end{array}$ & $0.361(0.230-0.567)$ & $0.411(0.259-0.654)$ & $0.472(0.293-0.762)$
\end{tabular}

Notes: adjusted model 1: adjusted for age, gender, vintage;

adjusted model 2: model $1+\mathrm{BMl}, \mathrm{Hgb}$, Alb, spKt/V, urine output, vascular access type;

adjusted model 3: model $2+$ comorbidities (diabetes, coronary artery diseases, congestive heart failure, other cardiovascular diseases, cerebrovascular disease, hypertension, peripheral vascular disease, hepatitis B and C, lung disease, cancer). 
Table 3

Associations between moderate activities limited level and first hospitalization in different COX regression models.

\begin{tabular}{|c|c|c|c|c|}
\hline & $\begin{array}{l}\text { Unadjusted } \\
\text { model } \\
\mathrm{HR}(95 \% \mathrm{Cl})\end{array}$ & $\begin{array}{l}\text { Adjusted model } 1 \\
\text { HR }(95 \% \mathrm{Cl})\end{array}$ & $\begin{array}{l}\text { Adjusted model } 2 \\
\text { HR }(95 \% \mathrm{Cl})\end{array}$ & $\begin{array}{l}\text { Adjusted model } 3 \\
\text { HR }(95 \% \mathrm{Cl})\end{array}$ \\
\hline \multicolumn{5}{|c|}{$\begin{array}{l}\text { Moderate activities limited } \\
\text { level }\end{array}$} \\
\hline $\begin{array}{l}\text { Yes, limited a } \\
\text { lot }\end{array}$ & Ref. & Ref. & Ref. & Ref. \\
\hline $\begin{array}{l}\text { Yes, limited a } \\
\text { little }\end{array}$ & $\begin{array}{l}0.763(0.632- \\
0.922)\end{array}$ & $0.841(0.672-1.052)$ & $0.906(0.716-1.146)$ & $0.954(0.753-1.207)$ \\
\hline $\begin{array}{l}\text { No, not } \\
\text { limited at all }\end{array}$ & $\begin{array}{l}0.564(0.437- \\
0.726)\end{array}$ & $0.658(0.499-0.867)$ & $0.720(0.555-0.935)$ & $0.747(0.570-0.978)$ \\
\hline \multicolumn{5}{|c|}{ Climbing stairs limited level } \\
\hline $\begin{array}{l}\text { Yes, limited a } \\
\text { lot }\end{array}$ & Ref. & Ref. & Ref. & Ref. \\
\hline $\begin{array}{l}\text { Yes, limited a } \\
\text { little }\end{array}$ & $\begin{array}{l}0.692(0.544- \\
0.880)\end{array}$ & $0.739(0.577-0.945)$ & $0.798(0.616-1.032)$ & $0.848(0.655-1.099)$ \\
\hline $\begin{array}{l}\text { No, not } \\
\text { limited at all }\end{array}$ & $\begin{array}{l}0.594(0.474- \\
0.746)\end{array}$ & $0.687(0.531-0.888)$ & $0.743(0.575-0.960)$ & $0.809(0.629-1.042)$ \\
\hline \multicolumn{5}{|c|}{$\begin{array}{l}\text { Notes: adjusted model 1: adjusted for age, gender, vintage; } \\
\text { adjusted model 2: model } 1+\text { BMI, Hgb, Alb, spKt/V, urine output, vascular access type; } \\
\text { adjusted model 3: model } 2+\text { comorbidities (diabetes, coronary artery diseases, congestive heart } \\
\text { failure, other cardiovascular diseases, cerebrovascular disease, hypertension, peripheral vascular } \\
\text { disease, hepatitis B and C, lung disease, cancer). }\end{array}$} \\
\hline
\end{tabular}

\section{Associations between climbing stairs limited level and outcomes}

Among 1254 patients with results of climbing stairs limited levels, 170 (1.4\%) of them died and 563 $(44.9 \%)$ had hospitalization records over the follow-up period. From the survival curves, we found that higher limited levels of climbing stairs had higher risk of all-cause mortality and hospitalization (log-rank test, $P<0.0001$, Fig. 1 C and D). In fully adjusted COX model, 'No, not limited at all' and 'Yes, limited a little' in climbing stairs were significantly associated with decreased risk of all-cause mortality compared with 'Yes, limited a lot' group (HR: 0.472, 95\% Cl:0.293-0.762 and HR: 0.574, 95\% Cl: 0.380-0.865, respectively) (Table 2, Fig. 2C). However, after adjusted for characteristics and comorbidities, neither 'no limited' nor 
'limited a little' in climbing stairs were significant with decreased risk of hospitalization (HR: $0.809,95 \%$ Cl: 0.629-1.042 and HR: 0.848, 95\% Cl: 0.655-1.099, respectively) (Table 3, Fig. 2D).

\section{Related factors of self-reported physical function}

We used the generalized linear mixed models to find the relationships among poor physical function and demographics, clinical data and comorbidities. Higher serum Alb was positively associated with better conditions in moderate activities, whereas older, female, longer dialysis vintage, catheter use, and history of coronary artery disease; cerebrovascular disease were related to higher limited in moderate activities ( $P$ $<0.05$, Table 4). Similar, older, female, longer dialysis vintage, catheter use, and history of diabetes, coronary artery disease, cerebrovascular disease were significantly associated with higher limited levels in climbing stairs $(P<0.05$, Table 4$)$. 
Table 4

Risk factors of lower self-reported physical function in China DOPPS5

\begin{tabular}{|c|c|c|c|c|}
\hline \multirow[t]{2}{*}{ Variables } & \multicolumn{2}{|c|}{$\begin{array}{l}\text { Moderate activities limited } \\
\text { level }\end{array}$} & \multicolumn{2}{|c|}{ Climbing stairs limited level } \\
\hline & OR $(95 \% \mathrm{Cl})$ & $P$ value & OR $(95 \% \mathrm{Cl})$ & $P$ value \\
\hline \multicolumn{5}{|l|}{ Demographics } \\
\hline Age (/1 year) & $\begin{array}{l}1.033(1.021- \\
1.046)\end{array}$ & $<0.0001^{\star}$ & $\begin{array}{l}1.039(1.027 \\
1.051)\end{array}$ & $<0.0001^{*}$ \\
\hline Sex (female vs. male) & $\begin{array}{l}1.786(1.256- \\
2.540)\end{array}$ & $0.0012^{\star}$ & $\begin{array}{l}1.822(1.328- \\
2.501)\end{array}$ & $0.0002^{*}$ \\
\hline Vintage (/1 year) & $\begin{array}{l}1.077(1.029- \\
1.127)\end{array}$ & $0.0014^{\star}$ & $\begin{array}{l}1.072(1.027- \\
1.119)\end{array}$ & $0.0015^{\star}$ \\
\hline $\operatorname{BMI}\left(/ 1 \mathrm{~kg} / \mathrm{m}^{2}\right)$ & $\begin{array}{l}0.988(0.948- \\
1.030)\end{array}$ & 0.5759 & $\begin{array}{l}1.011(0.964- \\
1.060)\end{array}$ & 0.6658 \\
\hline Urine output (yes vs. no) & $\begin{array}{l}0.697(0.484- \\
1.003)\end{array}$ & 0.0521 & $\begin{array}{l}0.720(0.502- \\
1.034)\end{array}$ & 0.0749 \\
\hline \multicolumn{5}{|l|}{ Laboratory tests } \\
\hline $\mathrm{Hgb}(/ 1 \mathrm{~g} / \mathrm{dl})$ & $\begin{array}{l}0.963(0.891- \\
1.041)\end{array}$ & 0.3432 & $\begin{array}{l}0.925(0.852- \\
1.004)\end{array}$ & 0.0621 \\
\hline Alb (/1 g/dl) & $\begin{array}{l}0.636(0.432- \\
0.936)\end{array}$ & $0.0218 *$ & $\begin{array}{l}0.726(0.492- \\
1.071)\end{array}$ & 0.1066 \\
\hline \multicolumn{5}{|l|}{ Dialysis prescription } \\
\hline spKt/V (/ 1 unit) & $\begin{array}{l}0.802(0.453- \\
1.418)\end{array}$ & 0.4470 & $\begin{array}{l}0.959(0.532- \\
1.731)\end{array}$ & 0.8901 \\
\hline Dialysis $<3$ times / week (yes vs. no) & $\begin{array}{l}1.062(0.717- \\
1.573)\end{array}$ & 0.7640 & $\begin{array}{l}0.839 \text { (0.559- } \\
1.260)\end{array}$ & 0.3969 \\
\hline $\begin{array}{l}\text { Vascular access (catheter vs. } \\
\text { Fistula) }\end{array}$ & $\begin{array}{l}2.629(1.639- \\
4.220)\end{array}$ & $<0.0001^{*}$ & $\begin{array}{l}1.869(1.113- \\
3.138)\end{array}$ & $0.0181 *$ \\
\hline \multicolumn{5}{|l|}{ Comorbidities (\%) } \\
\hline Diabetes (yes vs. no) & $\begin{array}{l}1.379(0.974- \\
1.951)\end{array}$ & 0.0698 & $\begin{array}{l}1.557(1.094- \\
2.217)\end{array}$ & $0.0140 *$ \\
\hline Coronary artery disease (yes vs. no) & $\begin{array}{l}1.761(1.194- \\
2.597)\end{array}$ & $0.0043^{*}$ & $\begin{array}{l}1.604(1.082- \\
2.377)\end{array}$ & $0.0185^{\star}$ \\
\hline Congestive heart failure (yes vs. no) & $\begin{array}{l}1.258(0.855- \\
1.850)\end{array}$ & 0.2432 & $\begin{array}{l}1.421(0.978- \\
2.066)\end{array}$ & 0.0657 \\
\hline $\begin{array}{l}\text { Other cardiovascular diseases (yes } \\
\text { vs. no) }\end{array}$ & $\begin{array}{l}1.192(0.717- \\
1.984)\end{array}$ & 0.4981 & $\begin{array}{l}1.066(0.687- \\
1.656)\end{array}$ & 0.7743 \\
\hline
\end{tabular}




\begin{tabular}{|c|c|c|c|c|}
\hline $\begin{array}{l}\text { Cerebrovascular disease (yes vs. } \\
\text { no) }\end{array}$ & $\begin{array}{l}1.998(1.218- \\
3.279)\end{array}$ & $0.0061^{\star}$ & $\begin{array}{l}1.908(1.165- \\
3.126)\end{array}$ & $0.0103^{*}$ \\
\hline Hypertension (yes vs. no) & $\begin{array}{l}0.867(0.540- \\
1.391)\end{array}$ & 0.5531 & $\begin{array}{l}0.858(0.569- \\
1.296)\end{array}$ & 0.4690 \\
\hline $\begin{array}{l}\text { Peripheral vascular disease (yes vs. } \\
\text { no) }\end{array}$ & $\begin{array}{l}1.523(0.712- \\
3.260)\end{array}$ & 0.2781 & $\begin{array}{l}1.963(0.959- \\
4.019)\end{array}$ & 0.0651 \\
\hline Hepatitis (yes vs. no) & $\begin{array}{l}0.941(0.594- \\
1.493)\end{array}$ & 0.7988 & $\begin{array}{l}0.815(0.494- \\
1.346)\end{array}$ & 0.4242 \\
\hline Lung disease (yes vs. no) & $\begin{array}{l}1.786(0.908- \\
3.510)\end{array}$ & 0.0927 & $\begin{array}{l}1.488(0.777- \\
2.850)\end{array}$ & 0.2302 \\
\hline Cancer (yes vs. no) & $\begin{array}{l}0.918(0.401- \\
2.100)\end{array}$ & 0.8400 & $\begin{array}{l}0.733(0.363- \\
1.479)\end{array}$ & 0.3857 \\
\hline
\end{tabular}

\section{Discussion}

We analyzed the associations between self-reported physical function and all-cause mortality and hospitalization in China DOPPS5, a large perspective cohort study of HD patients. The 2 items 'moderate activities limited level' and 'climbing stairs limited level' derived from the SF-12v2 reflected patient's physical function levels. We found that greater limitations in moderate activities were positively associated with all-cause mortality and hospitalization after adjustment for covariates. Meanwhile, higher limited levels of climbing stairs were significant associated with increased risk of all-cause mortality in fully adjusted COX model, whereas they weren't significant related to higher risk of hospitalization after adjusted for all covariates. We also found that several factors, such as older, female, longer dialysis vintage, lower serum Alb, catheter use and combining with diabetes, coronary artery disease, cerebrovascular disease were notably associated with higher limited levels of physical function in our patients.

Our results suggested that self-reported physical function may be a useful prognostic indicator when used alongside clinical risk factors for identifying patients who are at risk for adverse outcomes. Our results were consistent with previous studies, which found that poor physical function was associated with mortality and other events. Different methods have been used in studies to evaluate participants' physical function. Kathy et al explored that peak exercise oxygen uptake which reflected physical exercise capacity was a highly significant predictor of survival among $175 \mathrm{HD}$ patients[23]. In large cohort studies of HD patients, PCS score based on SF-12 or SF-36 were significantly associated with increased mortality [24, 25]. Jassal et al reported that physical dependence characterized by ability to perform activities of daily living (ADLs) and instrumental ADL (IADL) had strong dose-response associations with all-cause mortality in HD patients from 12 countries in the DOPPS [26]. Gait speed was a common test for physical performance tests, and Stephanie et al pooled results of 9 cohorts, found that gait speed was associated with survival in older adults[27]. Meanwhile, there were also several studies about 6-minutes walking test and muscle power and strength measurements in patients with chronic kidney disease[28, $29,13,30]$. 
To our knowledge, this was the first study that use 2 items in the SF-12 to measure self-reported physical function and exploration the relationship among physical function and clinical outcomes in a large cohort of HD patients. We hope that these findings could be helpful to giving some ideas for nephrologists and dialysis staff in improve patient care. First, self-reported physical function with just 2-items questions was a simple method to assess patients' physical function and could be routinely implemented in outpatient care. Second, measuring physical function in this way does not require the use of specific instruments and venues, which is a time-saving and economical method. Finally, nephrologists are usually the primary provider of exercise counseling for dialysis patients[31, 12]. Different studies have investigated that physical exercise significantly improves physical function and muscle strength[32,33]. The process of assessing physical function will deepen patient's awareness about improving physical function, thus becoming a motivator for doing exercise.

Our results shown that several factors could be modified to improve physical function level. Physical function may potentially be ameliorated by improving nutrition status in patients with hypoproteinemia or reducing the use of catheter and replace it with fistula appropriately. In addition, we need to pay more attention to patients who are older, with longer dialysis vintage or more comorbidities, because they are more likely to have poor physical function. Appropriate exercise interventions for these patients may help improve their physical function, thereby improving clinical prognosis[34, 35].

This study has several limitations. First, the self-reported physical function was based on the results of questionnaires. It may be more subjective and less accurate than other objective methods. Therefore, this may be a relatively simple method, but at the same time it also loses some precision or range of measurement. Second, we excluded some patients without answers of the questions about physical function. Although this may cause bias in the statistical results, the nonresponse rate was low, and we have fully adjusted for covariates to minimize the impact of this problem.

\section{Conclusions}

In conclusion, self-reported physical function was associated with all-cause mortality and hospitalization in Chinese HD patients. Assessment of self-reported physical function by 2 items of 'moderate activities limited level' and/or 'climbing stairs limited level' could be a simple and operative method in daily dialysis practice to improve patients' quality of life and outcomes. Further studies need to validate if corrections of modifiable risk factors of higher limited level of physical function, catheter use and low serum albumin, could improve patient's survival and minimize hospitalization.

\section{Abbreviations}

HD: Hemodialysis dialysis; CKD: chronic kidney disease; K/DOQI:Kidney Disease Outcomes Quality Initiative区HRQOL: health-related quality of life; PCS: Physical component summary; MCS: mental component summary; DOPPS: Dialysis Outcomes and Practices Pattern Study; BMI: body mass index; Hgb: hemoglobin; Alb: albumin; Kt/V: total urea clearance; HR: Hazard ratio; Cl: confidence intervals. 


\section{Declarations}

\section{Ethics approval and consent to participate}

The study was approved by the Ethics Committee of Peking University People's Hospital (ethical approval number: 2018PHB028-01). And all patients signed the written informed consent.

\section{Consent for publication}

Yes.

\section{Availability of data and materials}

The data used of this study are available from the corresponding author on reasonable request.

\section{Competing interests}

The authors declare that they have no relevant competing interests.

\section{Funding}

This article was supported by National Natural Science foundation of China, and the grant recipient is Li ZUO, grant number is 81870524 .

\section{Author Contributions}

Conception and design of research $₫$ Qingyu Niu, Xinju Zhao, Li Zuo, Fan Fan Hou

Analyzed data: Qingyu Niu, Xinju Zhao

Interpreted results of experiments: Qingyu Niu, Xinju Zhao, Li Zuo

Prepared figures: Qingyu Niu

Drafted manuscript: Qingyu Niu

Edited and revised manuscript: Liangying Gan, Fan Fan Hou, Xinling Liang, Zhaohui Ni, Xiaonong Chen, Yuqing Chen, Li Zuo

Approved final version of manuscript囚Li Zuo, Fan Fan Hou 


\section{Acknowledgments}

The Dialysis Outcomes and Practice Patterns Study (DOPPS) Program in China is supported by Vifor Fresenius Renal Pharma, Sanofi Renal, Nipro Trading (Shanghai) Co., Ltd, 3SBio Inc., B.Braun, CEMMA MEDICAL. All of them had no role in study design; collection, analysis, and interpretation of data; writing the report; and all support was provided without restrictions on publications.

\section{References}

1. Smyth A, Glynn LG, Murphy AW, Mulqueen J, Canavan M, Reddan DN, et al. Mild chronic kidney disease and functional impairment in community-dwelling older adults. Age Ageing. 2013 Jul;42(4):488-94.

2. Walker SR, Brar R, Eng F, Komenda P, Rigatto C, Prasad B, et al. Frailty and physical function in chronic kidney disease: the CanFIT study. Can J Kidney Health Dis. 2015;2:32.

3. Painter P, Clark L, Olausson J. Physical function and physical activity assessment and promotion in the hemodialysis clinic: a qualitative study. Am J Kidney Dis. 2014 Sep;64(3):425-33.

4. Clarkson MJ, Bennett PN, Fraser SF, Warmington SA. Exercise interventions for improving objective physical function in patients with end-stage kidney disease on dialysis: a systematic review and meta-analysis. Am J Physiol Renal Physiol. 2019 May 1;316(5):F856-f72.

5. Post A, Tsikas D, Bakker SJL. Creatine is a Conditionally Essential Nutrient in Chronic Kidney Disease: A Hypothesis and Narrative Literature Review. Nutrients. 2019 May 10;11(5).

6. Johansen KL, Chertow GM, Ng AV, Mulligan K, Carey S, Schoenfeld PY, et al. Physical activity levels in patients on hemodialysis and healthy sedentary controls. Kidney Int. 2000 Jun;57(6):2564-70.

7. Chen JL, Godfrey S, Ng TT, Moorthi R, Liangos O, Ruthazer R, et al. Effect of intra-dialytic, lowintensity strength training on functional capacity in adult haemodialysis patients: a randomized pilot trial. Nephrol Dial Transplant. 2010 Jun;25(6):1936-43.

8. Chudasama YV, Khunti KK, Zaccardi F, Rowlands AV, Yates T, Gillies CL, et al. Physical activity, multimorbidity, and life expectancy: a UK Biobank longitudinal study. BMC Med. 2019 Jun 12;17(1):108.

9. Nordstoga AL, Zotcheva E, SvedahI ER, Nilsen TIL, Skarpsno ES. Long-term changes in body weight and physical activity in relation to all-cause and cardiovascular mortality: the HUNT study. Int $J$ Behav Nutr Phys Act. 2019 May 20;16(1):45.

10. K/DOQI clinical practice guidelines for cardiovascular disease in dialysis patients. Am J Kidney Dis. 2005 Apr;45(4 Suppl 3):S1-153.

11. Painter P, Marcus RL. Assessing physical function and physical activity in patients with CKD. Clin J Am Soc Nephrol. 2013 May;8(5):861-72.

12. Johansen KL, Sakkas GK, Doyle J, Shubert T, Dudley RA. Exercise counseling practices among nephrologists caring for patients on dialysis. Am J Kidney Dis. 2003 Jan;41(1):171-8. 
13. Koufaki P, Mercer T. Assessment and monitoring of physical function for people with CKD. Adv Chronic Kidney Dis. 2009 Nov;16(6):410-9.

14. Koufaki P, Kouidi E. Current best evidence recommendations on measurement and interpretation of physical function in patients with chronic kidney disease. Sports Med. 2010 Dec 1;40(12):1055-74.

15. Woo J, Yau F, Leung J, Chan R. Peak oxygen uptake, six-minute walk distance, six-meter walk speed, and pulse pressure as predictors of seven year all-cause and cardiovascular mortality in communityliving older adults. Exp Gerontol. 2019 Sep;124:110645.

16. Moorthi RN, Avin KG. Clinical relevance of sarcopenia in chronic kidney disease. Curr Opin Nephrol Hypertens. 2017 May;26(3):219-28.

17. Ekinci C, Karabork M, Siriopol D, Dincer N, Covic A, Kanbay M. Effects of Volume Overload and Current Techniques for the Assessment of Fluid Status in Patients with Renal Disease. Blood Purif. 2018;46(1):34-47.

18. Lacson E, Jr., Xu J, Lin SF, Dean SG, Lazarus JM, Hakim RM. A comparison of SF-36 and SF-12 composite scores and subsequent hospitalization and mortality risks in long-term dialysis patients. Clin J Am Soc Nephrol. 2010 Feb;5(2):252-60.

19. Loosman WL, Hoekstra T, van Dijk S, Terwee CB, Honig A, Siegert CE, et al. Short-Form 12 or ShortForm 36 to measure quality-of-life changes in dialysis patients? Nephrol Dial Transplant. 2015 Jul;30(7):1170-6.

20. Ware J, Jr., Kosinski M, Keller SD. A 12-Item Short-Form Health Survey: construction of scales and preliminary tests of reliability and validity. Med Care. 1996 Mar;34(3):220-33.

21. Young EW, Goodkin DA, Mapes DL, Port FK, Keen ML, Chen K, et al. The Dialysis Outcomes and Practice Patterns Study (DOPPS): An international hemodialysis study. Kidney International. 2000;57:S74-S81.

22. Pisoni RL, Gillespie BW, Dickinson DM, Chen K, Kutner MH, Wolfe RA. The Dialysis Outcomes and Practice Patterns Study (DOPPS): design, data elements, and methodology. Am J Kidney Dis. 2004 Nov;44(5 Suppl 2):7-15.

23. Sietsema KE, Amato A, Adler SG, Brass EP. Exercise capacity as a predictor of survival among ambulatory patients with end-stage renal disease. Kidney Int. 2004 Feb;65(2):719-24.

24. Knight EL, Ofsthun N, Teng M, Lazarus JM, Curhan GC. The association between mental health, physical function, and hemodialysis mortality. Kidney Int. 2003 May;63(5):1843-51.

25. Mapes DL, Lopes AA, Satayathum S, McCullough KP, Goodkin DA, Locatelli F, et al. Health-related quality of life as a predictor of mortality and hospitalization: the Dialysis Outcomes and Practice Patterns Study (DOPPS). Kidney Int. 2003 Jul;64(1):339-49.

26. Jassal SV, Karaboyas A, Comment LA, Bieber BA, Morgenstern H, Sen A, et al. Functional Dependence and Mortality in the International Dialysis Outcomes and Practice Patterns Study (DOPPS). Am J Kidney Dis. 2016 Feb;67(2):283-92.

27. Studenski S, Perera S, Patel K, Rosano C, Faulkner K, Inzitari M, et al. Gait speed and survival in older adults. Jama. 2011 Jan 5;305(1):50-8. 
28. Painter P, Carlson L, Carey S, Paul SM, Myll J. Physical functioning and health-related quality-of-life changes with exercise training in hemodialysis patients. Am J Kidney Dis. 2000 Mar;35(3):482-92.

29. DePaul V, Moreland J, Eager T, Clase CM. The effectiveness of aerobic and muscle strength training in patients receiving hemodialysis and EPO: a randomized controlled trial. Am J Kidney Dis. 2002 Dec;40(6):1219-29.

30. Segura-Orti E, Kouidi E, Lison JF. Effect of resistance exercise during hemodialysis on physical function and quality of life: randomized controlled trial. Clin Nephrol. 2009 May;71(5):527-37.

31. Bender FH, Holley JL. Most nephrologists are primary care providers for chronic dialysis patients: results of a national survey. Am J Kidney Dis. 1996 Jul;28(1):67-71.

32. Manfredini F, Mallamaci F, D'Arrigo G, Baggetta R, Bolignano D, Torino C, et al. Exercise in Patients on Dialysis: A Multicenter, Randomized Clinical Trial. J Am Soc Nephrol. 2017 Apr;28(4):1259-68.

33. Jagannathan R, Ziolkowski SL, Weber MB, Cobb J, Pham N, Long J, et al. Physical activity promotion for patients transitioning to dialysis using the "Exercise is Medicine" framework: a multi-center randomized pragmatic trial (EIM-CKD trial) protocol. BMC Nephrol. 2018 Sep 12;19(1):230.

34. van Vilsteren MC, de Greef MH, Huisman RM. The effects of a low-to-moderate intensity preconditioning exercise programme linked with exercise counselling for sedentary haemodialysis patients in The Netherlands: results of a randomized clinical trial. Nephrol Dial Transplant. 2005 Jan;20(1):141-6.

35. Wang Y, Jardine MJ. Benefits of exercise training in patients receiving haemodialysis: a systematic review and meta-analysis. Br J Sports Med. 2011 Nov;45(14):1165-6.

\section{Figures}




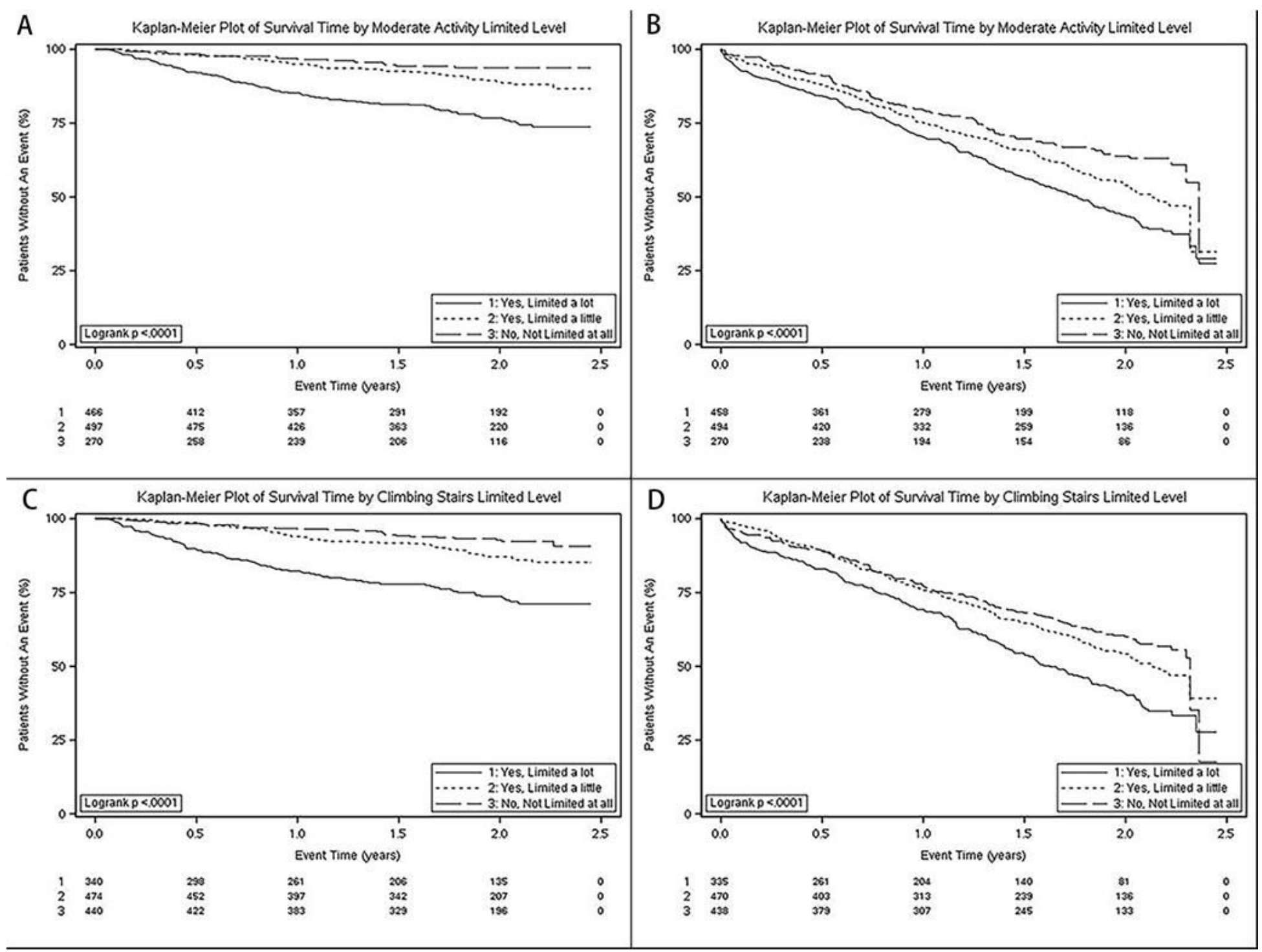

Figure 1

The Kaplan-Meier curves for different limited levels of self-reported physical function in HD patients. A, survival curves of all-cause mortality in different limited levels of moderate activities; B, survival curves of first hospitalization in different limited levels of moderate activities; $C$, survival curves of all-cause mortality in different limited levels of climbing stairs; $D$, survival curves of first hospitalization in different limited levels of climbing stairs. Log-rank test, $\mathrm{P}<0.001$ 

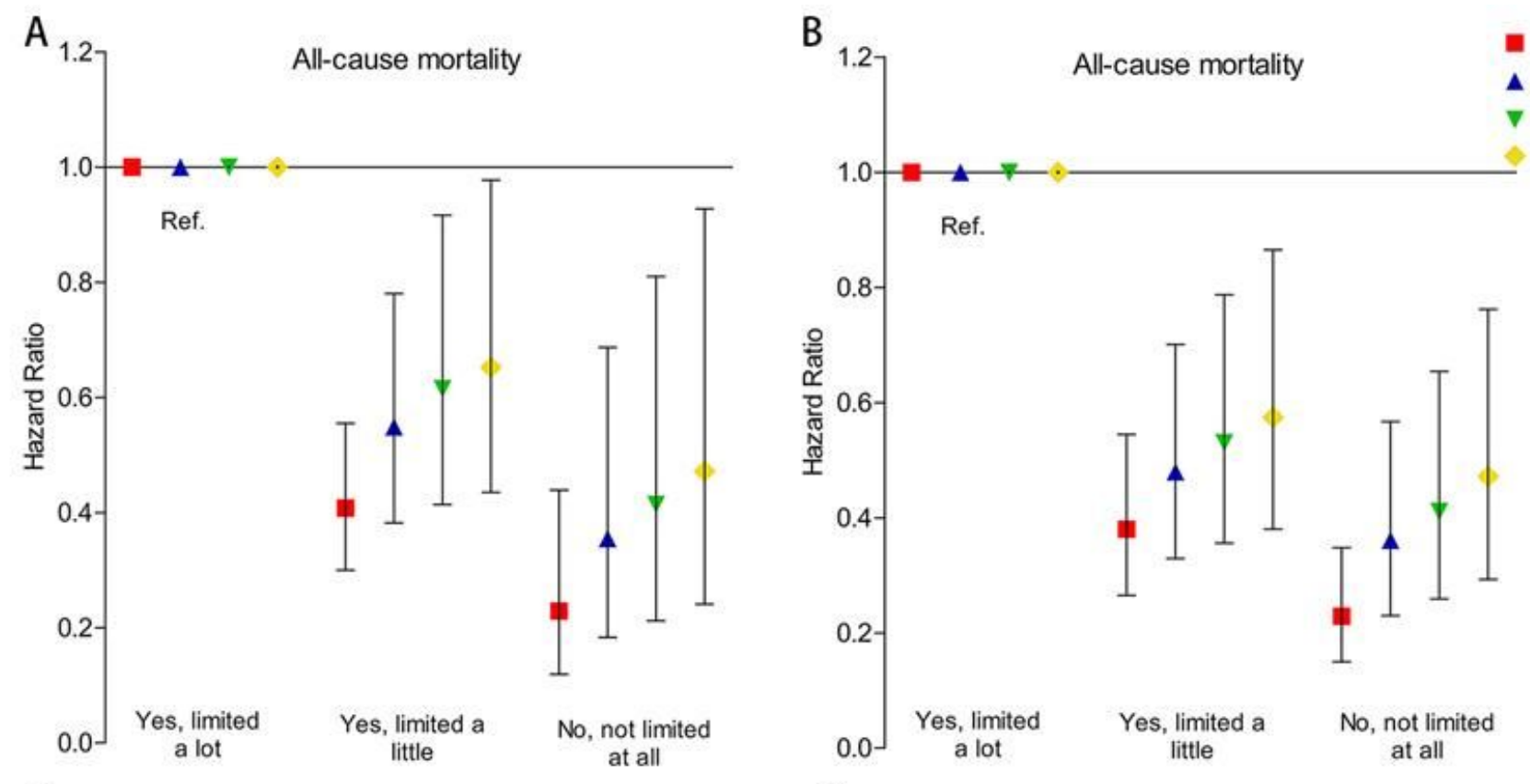

Unadjusted model Adjusted model 1 Adjusted model 2 Adjusted model 3
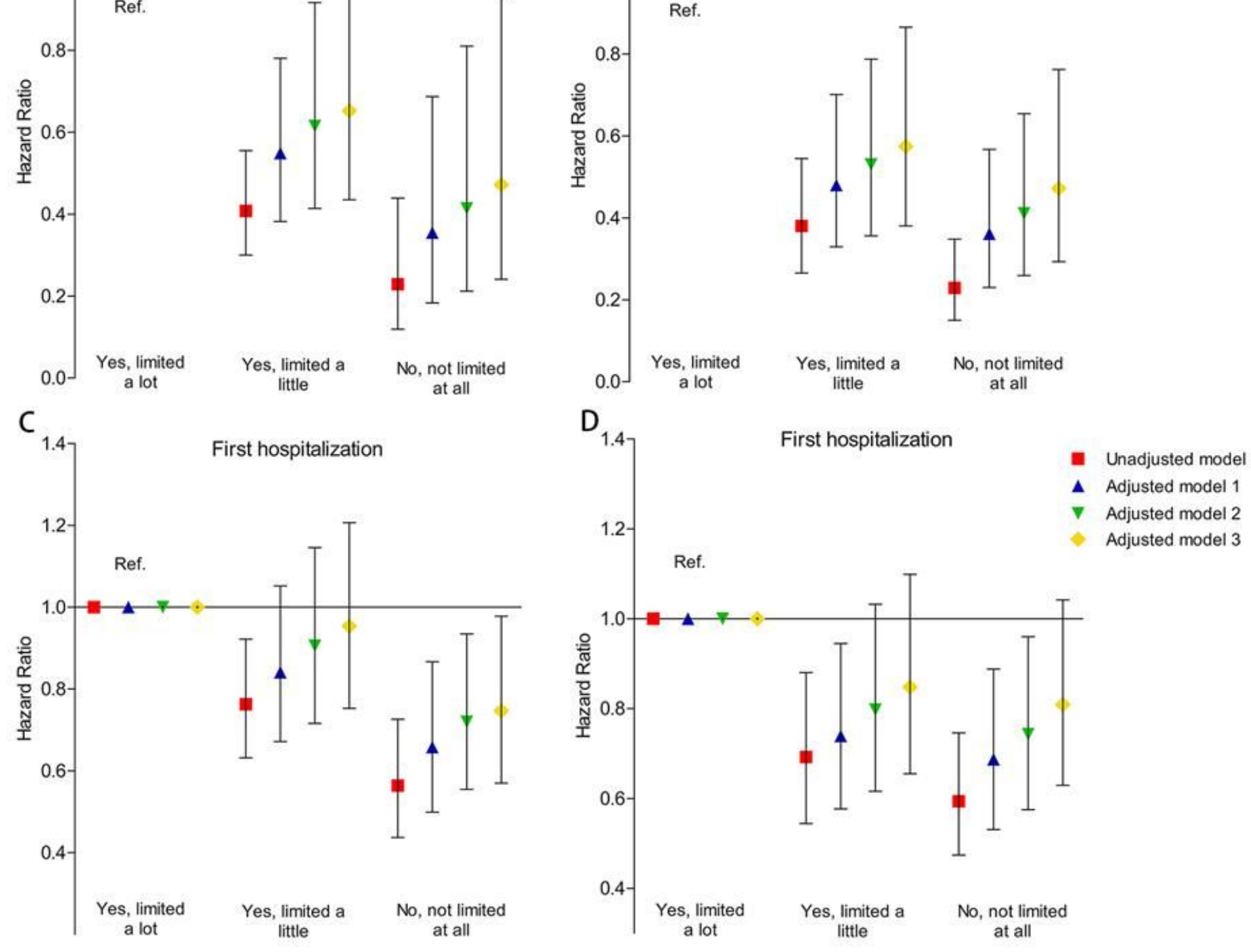

\section{Figure 2}

Associations between self-reported physical function and outcomes. A, associations between moderate activities limited level and all-cause mortality; $B$, associations between climbing stairs limited level and all-cause mortality; $\mathrm{C}$, associations between moderate activities limited level and first hospitalization; D, associations between climbing stairs limited level and first hospitalization. Adjusted model 1: adjusted for age, gender, vintage; adjusted model 2: model1 + BMl, Hgb, Alb, spKt/V, urine output, vascular access type; adjusted model 3: model 2 + comorbidities (diabetes, coronary artery diseases, congestive heart failure, other cardiovascular diseases, cerebrovascular disease, hypertension, peripheral vascular disease, hepatitis B and C, lung disease, cancer).

\section{Supplementary Files}


This is a list of supplementary files associated with this preprint. Click to download.

- Supplementarydata.docx 